\title{
Necrotising pulmonary aspergillosis complicating the management of patients with obstructive airways disease
}

\author{
PJM GEORGE, PBJ BOFFA, CPE NAYLOR, TW HIGENBOTTAM \\ From the Thoracic Medical Unit, Papworth Hospital, and the Department of Histopathology, New \\ Addenbrooke's Hospital, Cambridge
}

Necrotising or invasive aspergillosis is a rare condition which occurs predominantly in immunocompromised patients. $^{1}$ It is a major cause of death in some groups of patients, notably in acute leukaemia. ${ }^{2}$ We describe two cases of necrotising pulmonary aspergillosis in patients with obstructive airways disease in whom the diagnosis was made only at necropsy.

\section{Case reports}

\section{Patient 1}

A 60-year-old man with an eight-year history of late-onset asthma was admitted to hospital with severe bronchoconstriction, which had persisted despite outpatient treatment

Address for reprint requests: Dr Peter Boffa, Department of Thoracic Medicine, Papworth Hospital, Cambridge CB3 8RE.

Accepted 19 November 1982

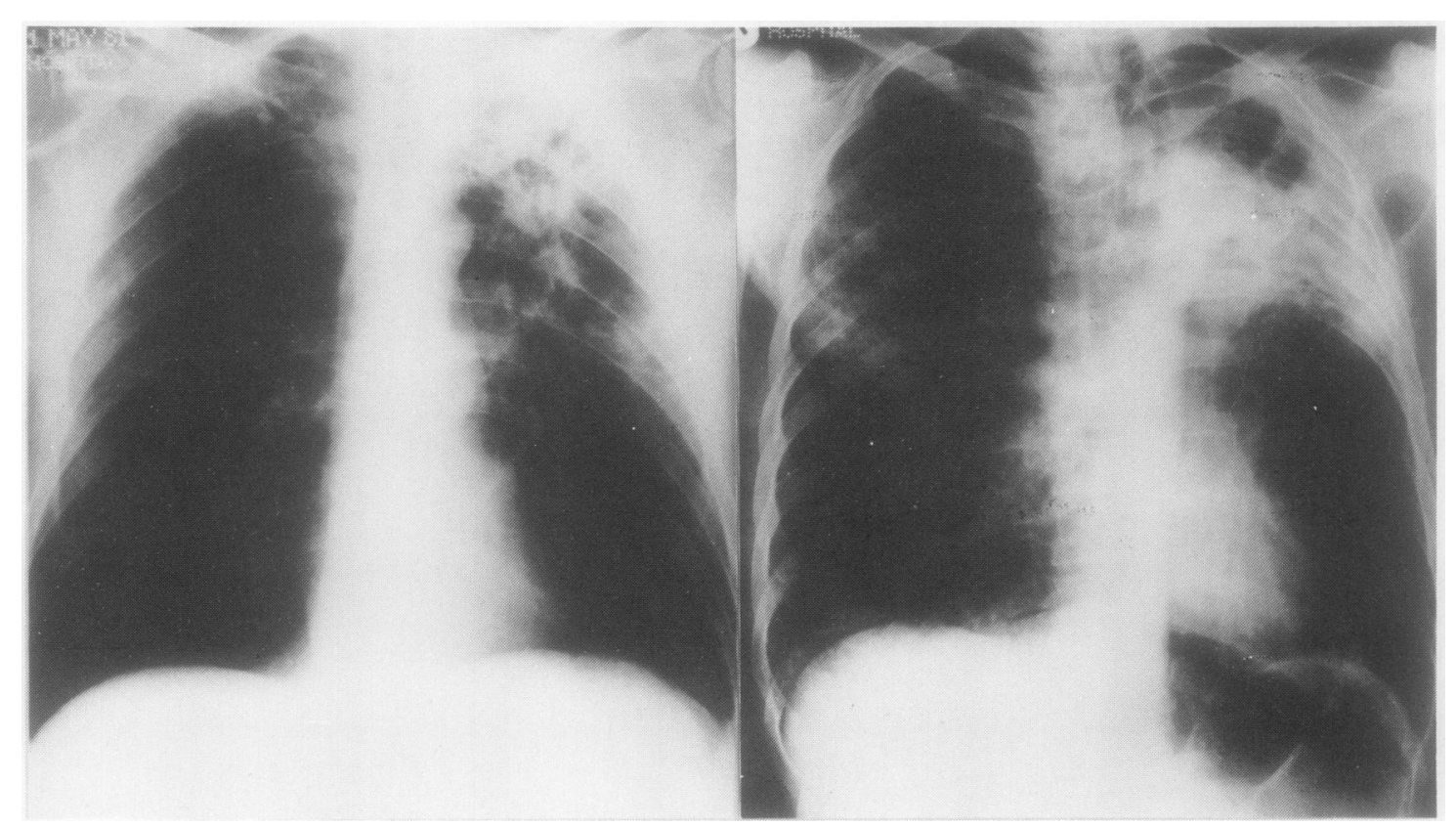

Fig 1 Chest radiographs from case 1 (left) and case 2 (right). with prednisolone (30 $\mathrm{mg}$ daily). The chest radiograph showed hyperinflated lung fields with a left apical bulla, treated with nebulised bronchodilators, intensive physiotherapy, and oral prednisolone in the same dosage. After two weeks there had been little change in his condition and the dose of prednisolone was increased to $60 \mathrm{mg}$ daily. There was no improvement and after 10 days he developed purulent sputum, which grew Streptococcus pneumoniae. The dose of prednisolone was reduced to 30 mg daily and oral ampicillin was added. Despite antibiotic treatment his condition slowly deteriorated and after five weeks left upper-lobe consolidation became apparent on the chest radiograph (fig 1). His white blood count was raised to $20 \times 10^{9} / 1$ with $90 \%$ neutrophils. Serum immunoglobulins were within normal limits and sputum culture showed Klebsiella pneumoniae, which was resistant to gentamicin. Aspergillus precipitins, measured by counterimmunoelectrophoresis, were not detected in serum and aspergillus hyphae were not found in the sputum. He which had been noted on previous radiographs. He was 


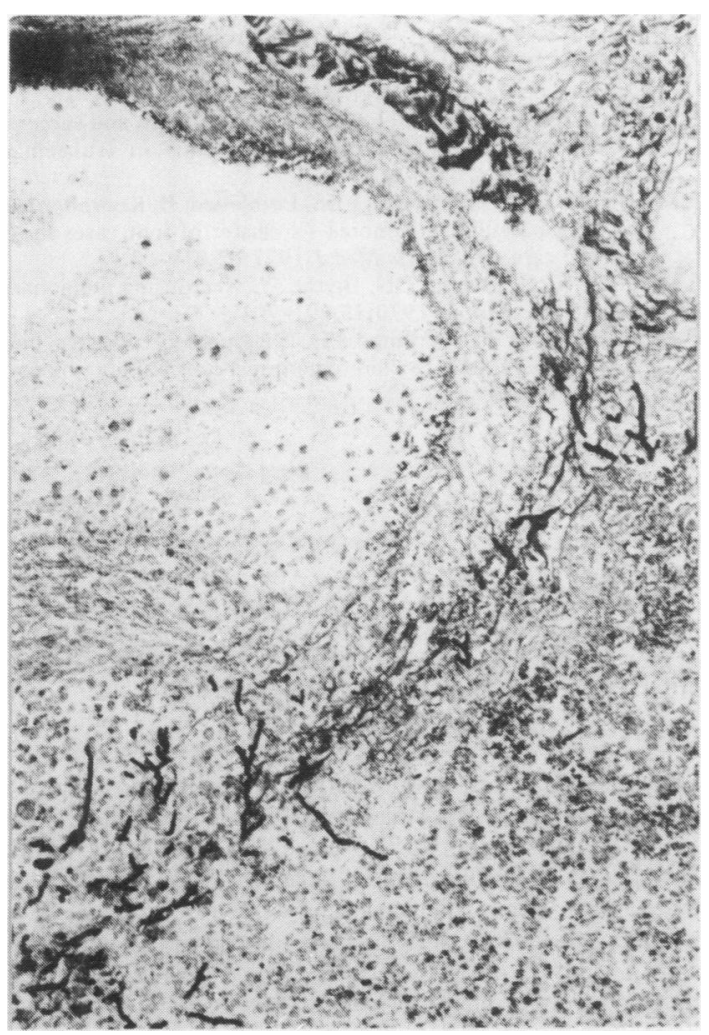

Fig 2 Small pulmonary artery from right middle lobe of case 1: Grocot's silver method shows invading fungal hyphae $(\times 125)$.

continued to deteriorate despite treatment with cefotaxime, and developed respiratory failure necessitating artificial ventilation. Twelve days later he suffered a cardiac arrest and died after a total period of 14 weeks in hospital.

Postmortem examination of the lungs showed a densely adherent $10-\mathrm{cm}$ cavitating lesion at the left apex lined by fungal colonies. Aspergillus fumigatus was cultured from the contained mycetoma. Both lungs were severely emphysematous and bullae were present. There was widespread fungal pneumonia with haemorrhage, necrosis, and abscess formation. Fungal hyphae were seen invading blood vessel walls (fig 2). There was no evidence of old or active tuberculosis. Examination of the kidneys showed a focus of necrotising papillitis invaded by aspergillus hyphae, confirming dissemination.

\section{Patient 2}

A 55-year-old man, who had previously been diagnosed as suffering from chronic bronchitis and emphysema, was admitted to hospital for investigation of a recently acquired radiographic shadow in the left upper lobe. Rigid bronchoscopy showed no endobronchial lesion and the aspirate from the left upper lobe contained no malignant cells or potential pathogens. Three days after bronchoscopy he became acutely ill with severe bronchoconstriction and clinical and radiographic signs of a left upper-lobe pneumonia. Full blood count, urea and electrolyte concentrations, liver function test values, and blood sugar and serum immunoglobulin concentrations were within normal limits. Aspergillus precipitins were not detected in the serum. He was treated with oral amoxycillin, flucloxacillin, prednisolone $(20 \mathrm{mg}$ daily), and inhaled bronchodilators but developed new, ill-defined shadowing in the right upper lobe (fig 1). Sputum and blood cultures were repeated but gave negative results. The antibiotic treatment was changed to intravenous ampicillin and gentamicin, but his condition deteriorated and he died 20 days after admission.

Postmortem examination confirmed the presence of severe emphysema in both lungs and showed old organised pneumonic consolidation in the left upper lobe. Colonies of Aspergillus sp were found in extensive areas of necrotising bronchitis in the right upper and lower lobes. There was no evidence of malignant disease or of tuberculosis.

\section{Discussion}

The two cases reported here illustrate some of the difficulties of diagnosing necrotising aspergillosis in life. Although both patients had widespread fungal pneumonia, repeated sputum and blood cultures failed to grow aspergillus and precipitating antibodies could not be detected in the serum. Such findings are not exceptional in patients with known necrotising infections. ${ }^{34}$ The discovery of positive sputum cultures ${ }^{5}$ or of aspergillus precipitins would have raised the possibility of a necrotising infection but these are also consistent with saphrophytic colonisation of the respiratory tract. ${ }^{\circ}$

Despite these limitations, non-invasive investigations are frequently used to diagnose necrotising aspergillosis in patients with leukaemia, who are known to be particularly susceptible.' It has recently been argued that any patient who has hepatic, renal, or respiratory insufficiency and the features of a generalised infection should be considered for antifungal treatment if the tracheal secretions contain aspergillus. $^{5}$ Although cases of necrotising pulmonary aspergillosis have been described in patients with obstructive airways disease ${ }^{89}$ the association is uncommon and we suggest that firm evidence of lung invasion by aspergillus hyphae is desirable before such patients are committed to potentially toxic treatment. In the absence of more reliable non-invasive methods of investigation, fibreoptic transbronchial lung biopsy may provide the best method of obtaining such a diagnosis. ${ }^{10}$

The use of antibiotics and corticosteroids in patients with pre-existing lung disease has been implicated as a predisposing factor in opportunistic fungal pneumonia,' and may have contributed to the two cases reported here. Our experience emphasises the need to consider the possibility of necrotising pulmonary aspergillosis in similar clinical circumstances, and shows the inadequacy of non-invasive methods of investigation.

We would like to thank Dr JE Stark for allowing us to report details of his patients and Dr PGI Stovin for performing one of the postmortem examinations. 


\section{References}

' Young RC, Bennet JE, Vogel CL, Carbone PP, De Vita VT. Aspergillosis: the spectrum of disease in 98 patients. Medicine (Baltimore) 1970;49:147-73.

${ }^{2}$ Mirsky HS, Cuttner J. Fungal infection in acute leukaemia. Cancer 1972;30:348-52.

${ }^{3}$ Meyer RD, Young LS, Armstrong D, Yu B. Aspergillosis complicating neoplastic disease. Am J Med 1973;54:6-15.

4 Young RC, Bennet JE. Invasive aspergillosis? absence of detectable antibody response. Am Rev Respir Dis 1971;104:710-6.

5 Park GR, Drummond GB, Lamb D, et al. Disseminated aspergillosis occurring in patients with respiratory, renal and hepatic failure. Lancet 1982;ii:179-83.

\section{Notices of books received}

Cash's Textbook of the Chest, Heart and Vascular Disorders for Physiotherapists. Ed Patricia A Downie. (Pp 493; £7.50.) Faber and Faber. 1983.

The third edition of this textbook for physiotherapists has been radically revised from the previous edition with many new authors. It maintains the high standards set by its predecessors and is clear in its descriptions of fairly complex physiology and physics. It covers basic anatomy and physiology, clinical examination, chest radiography, lung function tests, and clinical aspects of intensive care, cardiothoracic surgery, medical chest disease, myocardial infarction, and common vascular disorders. It is likely to be a bestseller as its predecessors were and will be a useful book not only for physiotherapists but also for nurses and junior medical staff.

Topics in Gastroenterology 10. Ed DP Jewell, WS Selby. (Pp 337; £20.) Blackwell Scientific Publications. 1982.

This is the tenth volume entitled Topics in Gastroenterology. It is based on papers presented at the postgraduate gastroenterology course held in Oxford in January 1982. The main topics covered are oesophageal carcinoma, oesophageal motility, peptic ulcer, the short gut, cholestasis, paediatric gastroenterology, the absorption of vita$\min B_{12}$, and the aetiology of Crohn's disease. The first five chapters, on the oesophagus, are the only ones relevant to thoracic physicians and surgeons, and there is an excellent discussion of the epidemiology of oesophageal carcinoma by Sir Richard Doll. The chapters on the physiology of the oesophagus and assessment of oesophageal function are clear and make the relatively new science of oesophageal function studies easy to understand.
- Strimlan CV, Dines DE, Rodgers-Sullivan RF, Roberts GD, Sheehan WC. Respiratory tract Aspergillus: clinical significance. Minn Med 1980;63:25-9.

${ }^{7}$ Sinclair AJ, Rossof AH, Coltman CA. Recognition and successful management in pulmonary aspergillosis in leukaemia. Cancer 1978;42:2019-24.

- Kallenbach J, Dsheiko J, Block CS, Bethlehem B, Koornhof HJ, Zwi S. Aspergillus pneumonia-a cluster of four cases in an intensive care unit. $S$ Afr Med J 1977;52:919-23.

${ }^{9}$ Kennedy WPU, Malone DN, Blythe W. Necrotising pulmonary aspergillosis. Thorax 1970;25:691-701.

${ }^{10}$ Chung C, Lord PL, Krumpe PE. Diagnosis of invasive pulmonary aspergillosis by fibreoptic transbronchial lung biopsy. JAMA 1978;239:749-50.

Thoracic and Cardiovascular Surgery. William WL Glenn, Arthur E Baue, Alexander S Geha, Graeme L Hammond, Hillel Laks. (Pp 1667; \$125.) Appleton-Century-Crofts, Norwalk, Connecticut. 1983.

Although this is called the fourth edition of Thoracic and Cardiovascular Surgery it is such a complete revision that it is really a new textbook. There are 114 chapters covering all aspects of cardiothoracic surgery. As is usual in a multi-author book, the clarity and value of each chapter is variable, but the line drawings and photographs are of high quality. It is well indexed and each chapter contains a list of useful references, many of which for a volume of this size are reasonably up to date. The book is a useful addition to the standard reference works in cardiothoracic surgery and all medical libraries and most specialist surgical libraries should have a copy.

Handbook of Intensive Care. Ed WH Bain, KM Taylor. (Pp 564; £35.) John Wright and Sons. 1983.

This new handbook is intended to provide a review of, and a guide to, modern intensive care. The chapters are written by 29 invited contributors, of whom 24 are from Scotland, two from the United States, one each from India, Belfast, and Newcastle upon Tyne. The topics covered are divided into those which relate to the subject or the patient as a whole (for example, logistics of intensive care, monitoring, fluid balance, and treatment) and those where individual systems are considered (for example, respiratory, cardiovascular, renal). As with any multi-author work there are some areas of overlap and some omissions and the text is in some areas trite and in others excessively detailed. Nevertheless, the work will provide a useful reference guide to clinicians who are concerned with this area of medicine. 